\title{
Stakeholders Perception of Current Health Education Situation under Ghana's Health Service
}

\author{
K. B. Antwi \\ Department of Geography and Tourism, University of Cape Coast, Cape Coast, Ghana \\ Present address: Centre for International Development (BCID), University of Bradford, Bradford, West \\ Yorkshine BD 7, 7DP, U.K. \\ Corresponding author; E-mail: k_bantwi@yahoo.co.uk
}

\begin{abstract}
Health Education is one of the critical eight essential pillars of the primary health care (PHC) adopted world-wide by WHO member countries in 1978. After over two decades of health education to support PHC implementation, the epidemiological profile of Ghana continues to be dominated by communicable diseases, and environmental sanitation is problematic in both urban and rural areas. Based on the health field concept, the behavioural and information diffusion theory, the paper provides a situational overview of health education in Ghana. Informants were health policy makers, managers, healthcare providers and the public, as they perceive health education delivery in healthcare institutions, schools, workplaces and communities. Quantitative and qualitative techniques including questionnaires, semi-structured interviews and focus group discussions were employed to reach the target population. The paper concludes that despite its importance health education seemed to enjoy less support at policy, management, service providers and users of healthcare levels. However, it is a worthwhile and cost effective strategy that must be nurtured towards health promotion.
\end{abstract}

\section{Introduction}

One of the outstanding demands of the English Public Health Movement of the 19th Century was for the improvement of the physical environment. Chadwick, one of the disciples of the movement, argued that 'better sanitation is a good investment and prevention of diseases could offer greater benefit than the building of hospitals to treat those diseases'. (Lee \& Mills, 1983), These lofty ideas have obvious similarities to contemporary ideas on health strategies and on the importance of health improvement in developing countries in particular.

The international consensus on policy shifts in health and development came in 1987, at a joint World Health Organization (WHO) and United Nations Children Fund (UNICEF) conference on Primary Health Care (PHC) at Alma Ata, USSR. It was here that the 'imbalances in health resource allocation' such as situations where as much as $80 \%$ of national health expenditure might be used for large hospitals, which are accessible to less than $20 \%$ of the population, was recognized (M'Jamtusie, 1996)

A broader re-definition of health was, therefore, adopted as 'a state of complete physical, mental and social well-being and not merely the absence of diseases or infirmity' (WHO, 1986). It was further an encouragement that the Alma Ata Conference asserted the 'centrality of health education' as the first of the essential strategies for achieving health-for-all by the year 2000 (HFA 2000). The importance of health education towards health improve-ment was realized as early as 1943 when the maiden edition of Health Education Journal was launched in the United Kingdom. The Minister of Health, Ernest Brown, was reported to have recalled that:

'One of the most noticeable characteristics of the dark ages was fatalism; men were overwhelmed by circumstances; they existed under a sense of impending disasters that they could neither see nor prevent. Hence education is the instrument of reform, the giver of hope, the guide which dissects the conscious individual effort without which health cannot be attained'. (Tones \& Tilford, 1994).

The PHC basic strategy included the 'medical model', but not just medical diagnosis and treatment dealing with individual sickness and cure. This is not to belittle the importance of medical interventions for individuals or to suggest that medical care should be relegated to the background (Collins, 1994). What it does suggests, however, is that health policy would do well to pay particular attention to the underlying causes of ill-health and also focus on 'preventive' and 'promotive' activities, such as the

West African Journal of Applied Ecology - Volume 13 
improvement in water supply and sanitation, housing, nutrition, education and employment, which can have a significant impact on health.

Unfortunately, the situation in Sub-Sahara Africa is quite difficult, and most worrying because there are many health problems still to be solved due to what Hubert (1994) has described as 'epidemics of ignorance'. On this sub-continent, diarrhoea ailments cause $38 \%$ of fatalities among children less than 5 years of age, despite the fact that simple oral re-hydration therapy could considerably reduce the risk of death. These diseases take a greater toll because of 'lack of information (Collins, 1994).

Apart from these, the region has been plagued with multi-facet problems ranging from political (civil wars and coup d'etat), economic (declining incomes), socio-cultural and environmental; which have negatively impacted on the health care system. Kunfah (1996) has conceptualized these as some kind of crisis factors with a close relationship to health and welfare (Fig. 1). One can observe the complex, relationships between those factors and the provision of health services; be it access, coverage, quality and financing.

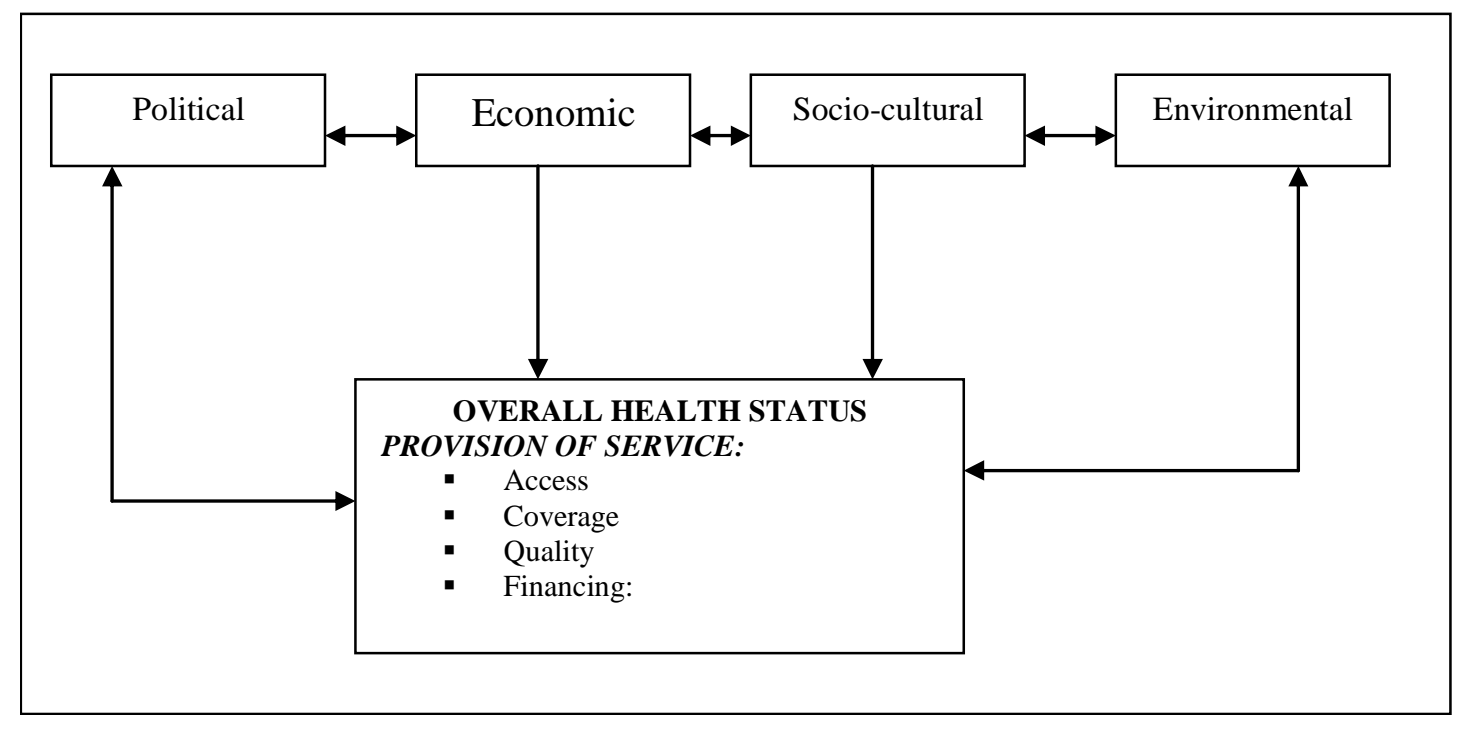

Source: Kunfah, 1996

Fig. 1. Relationships between Socio-Economic Crisis and Health

Various world development reports produced in the last decade have tended to show that the economic crisis in particular, and its attendant poverty, both absolute and relative, have been found to influence the actions of individuals and groups to risk taking and health seeking behaviour in general (Awusabo-Asare \& Anarfi, 1999). These observations, among others, tend to suggest that health education has been somehow marginalized in later developments as one of the 'essential strategies' to better health, probably due to the 'selective' versus 'comprehensive' PHC policy debates of the late 1980's (Asamoah-Baah, 1990).

Kok \& Green (1990) have observed with concern about the act of co-operation between researchers and professionals, and have argued that health promotion professionals and administrators are not always interested in, or receptive to, evaluation research. It could be said with some justification that the task of education is to safeguard people's rights and empower them to learn about important aspects of human culture and experience. Since health and illness occupy a prominent place in everyday experience, it might reasonably be argued that everyone is morally entitled to share whatever insights health authorities and professionals possess about the 'state of being healthy' and to benefit from what might be done to West African Journal of Applied Ecology - Volume 13 
prevent and treat diseases and discomfort. The role of health education in such an endeavour would be to create the necessary understanding of which a credible mandate has been given, since Ghana adopted the PHC policy strategy in 1978.

In recent years, however, public health and social policy advocates have questioned with increasing insistence about effectiveness, efficiency and equity, both about education in general and health education in particular. These enquiries reflect, to a greater extent, as to whether or not the Ghanaian population is better informed on health issues, given the increasing demand for services, on one hand, and relative shrinking of health resources, on the other. It is against this background that this paper seeks to examine perceptions of the various stakeholders' current health education situation under Ghana's HPC program. The purpose of the paper is to contribute to the policy debate on the need to emphasize preventive health, despite the fact that it takes time to realize its benefits.

The origin of health promotion (HP) may be found in WHO's original conception of health (WHO, 1984), with its holistic emphasis of the positive. The 'health field concept' which was popularized by Lalonde (1974), on the health of Canadians, set out to identify four crucial factors which influence health, viz: 'Socio-economic, cultural and physical environment, genetic (biology), individual lifestyles and health services' (Tones \& Tilford, 1994).

Given that the genetic aspect of this model is not effectively amendable to intervention (except perhaps by genetic counseling), the concerns of health promotion would in practice centre on the remaining three factors. However, as a result of the exhortation to re-orient health (medical) services, curative medicine has often been excluded from health promotion field, leaving lifestyle and the environment as the main areas of interest (Tones \& Tilford, 1994). This re-thinking was further strengthened when WHO accepted that health promotion is 'a unifying concept for those who recognized the need for change in the ways and conditions of living, in order to promote health' (WHO, 1984). As a result of the debate on the desirability of professionalizing health promotion, a wide variety of different interest groups has laid claim to this essentially contested concept (Green \& Raebuun, 1988).

The ideological basis of the WHO formulation of health promotion is evident in the Ottawa Charter (WHO, 1986) and associated publications and pronouncements. The ethical and moral views of humanity enshrined in this perspective are many and couched in principles and statements of beliefs (WHO, 1984). Two principles relevant for this paper are that (1) Health should be viewed holistically as a positive state; it is an essential commodity which people need in order to achieve the ultimate goal of a socially and economically productive life; and (2) Health is too important to be left to medical professionals alone; there must be a 're-orientation of health services'. Because medical services frequently do not meet the needs of the public; the professionals often treat people as passive recipients of health care and are, thus, fundamentally de-powering and victim blaming.

The WHO Ottawa Charter (1986), defined health promotion as 'the process of enabling people to increase control over and improve their health'. In a critical analysis of the WHO definition, Kelly (1990), while recognizing its practical implications, is disturbed by the lack of clarity. Firstly, the difference between the medical and social models of health and illness which stress that high technology medicine is said to encourage dependency on the medical profession and discharge individuals and groups from taking responsibility for their own health does not manifest. Secondly, the definition's strong orientation towards action for change for the better, which he termed 'target setting', is misleading. Because there is the danger that targets for future achievement may distract attention from present health promotion needs in existing communities. He, therefore, concluded that 'health promotion should not be portrayed as an exclusive alternative to medicine, but must work with it towards understanding the origins of health in addition to abuses of diseases'.

Agreeing with Kelly (1990), Green \& Raeburn (1988) are convinced of the added scope and purpose of health promotion, but feat that bureaucrats and politicians who formulate and carry out healthy promotion policies in their countries might quietly ignore the concept.

West African Journal of Applied Ecology - Volume 13 
Health promotion has also been explained by Ewles \& Simnett (1992), using five integrated elements to suggest that there is no one 'right' approach to health but multiple, including (1) Medical-which aims at freedom from medically defined disease and disability; (2) Behavior change - that ensures that individual behavior is conducive to freedom from disease. (3) Educational - provides individuals with knowledge and understanding, enabling them to take well-informed decisions and act upon them; (4) Client centered - which aims at working with clients on clients own terms; and (5) Societal change - works on the physical and social environment that enables choice of healthier lifestyle.

Tannahill et al. (1990), introduced a simplified combinational approach to health promotion quite similar to Ewles \& Simnett (1992). They defined health promotion as comprising 'efforts to enhance positive health and prevent ill-health through the overlapping spheres of health education, health prevention and health protection'. The model works in an integrated fashion with the three strategies intersecting in a form of Venn diagram to generate four additional areas of cooperation to achieve unity of purpose in health promotion as illustrated in Fig. 2.

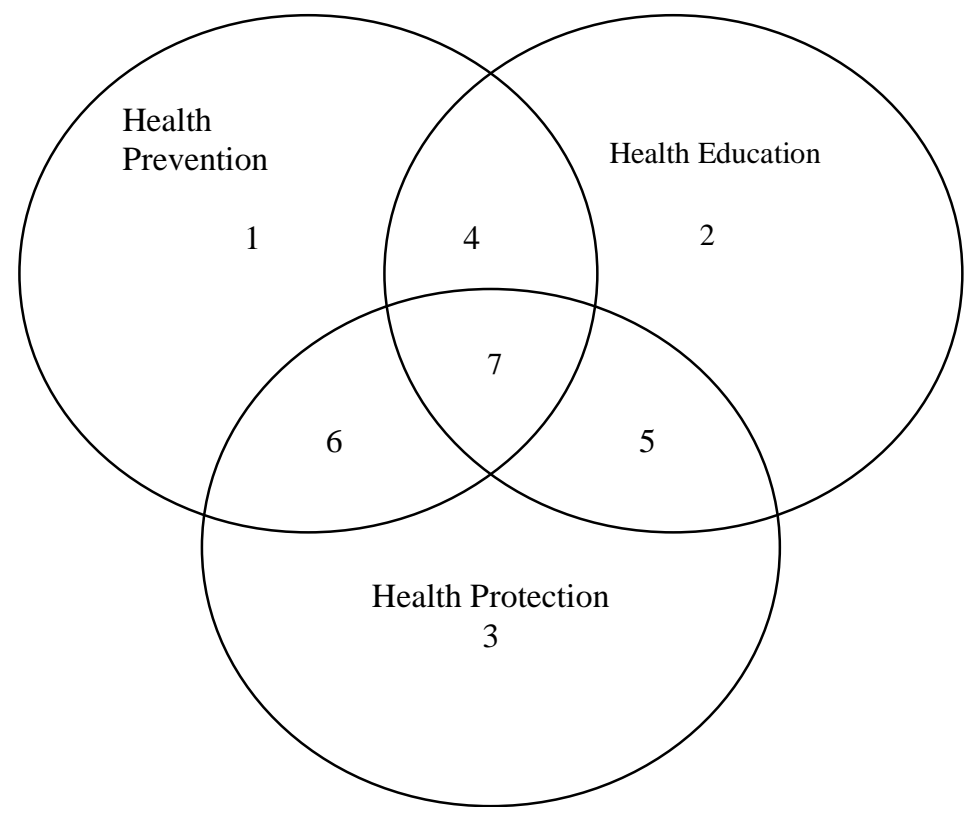

Key

1. Positive Health Prevention

2. Positive Health Education

3. Positive Health Protection

4. Preventive Health Education

5. Health Education Aimed at Positive Health Protection

6. Preventive Health Protection

7. Health Education for Preventive Health Protection

The Tannahill model and the seven domains it presents suggest empowerment as the cardinal principle of health promotion. This means health education seeks to empower by providing the necessary information and helping people to develop skills and an acceptable level of self-esteem, so that they come to feel that significant control resides in them, rather than feeling threatened by external forces. Thus, the provision of good preventive services and the shaping of a healthful environment through health protection also contribute to this empowerment process (Friedman, 1992).

West African Journal of Applied Ecology - Volume 13 
The implicit assumption underlying health education is that it is a worthwhile activity and, in some way, good for the public, but the term means different things to different people. It may mean public relations aimed at publicizing the activities of health departments and winning public goodwill, since many people tend to equate it with the transmission of information about health and disease from expert professionals to the lay clients (Collins, 1994; Dennis, 1992). These perceptions may be attributable to both the historical development of health education and an understanding of the learning process.

Tones \& Tilford (1994) have defined health education as 'Any intentional activity which is designed to achieve health or illness related learning: Effective health education may, thus, produce changes in knowledge and understanding of ways of thinking; it may influence or clarify values; it may bring about some shift in belief or attitude; it may facilitate the acquisition of skills; it may even effect changes in behavior of life style'. Similarly, Pisharoti (1975) cited by Tones \& Tilford (1994) described it as 'a process leading to programmed planning, utilizing available resources, modifying health behavior, breaking down barriers of ignorance, prejudice and misconceptions after an intelligent and thoughtful consideration of relevant health knowledge. It is a translation of what is known about health into desirable, individual, family and community behavior patterns by means of an educational process'.

The change in scope of health education and the symbolic relationship between health education and promotion, as established by Tannahill et al. (1990), Tones \& Tilford (1994) and Pisharoti's (1975) conception of the contribution of health education to health promotion, is worth examining. For policy and practical reasons, two strategies for producing change tend to emerge from these views, namely 'lobbying and education'. Of the two, the latter is considered to have potentially more powerful and multifaceted role. Lobbying may, nonetheless, make a major contribution to health promotion, and its function is primarily helping to bring about appropriate public health policy. Thus, both education and policy are central to the achievement of individuals, community and national health status.

The policy and practical implications of the issues raised are that health education tends to have fourfold function that interrelates to enhance health promotion. One of these relates to individuals decisionmaking; a second concerns the development and appropriate use of health services; and the last two, i.e. lobbying and advocacy, relate to facilitating the formulation and implemen-tation of a responsible public health policy, through mediation and dialogue. In reality, therefore, health education enhances the use of learning experiences designed to facilitate voluntary adaptation of behavioral concerns to health. Understanding the education of individuals, households, and the entire community calls for the application of a mixture of different approaches in a framework that ensure the realization of health education goals and health promotion in particular. Given that health is substantially influenced by environmental factors that may be positive or negative, the paper adopts a multi paradigm framework to attempt an explanation of the health education situation in Ghana as perceived by the various stakeholders.

The Health Field Concept (Lolande, 1974), Preds Behavioral Model (Johnston, Gregory \& Smith, 1998) and Haggestrands Information Diffusion Model (Gregory, 1985; Hagget \& Chorley, 1968) provide the theoretical basis for this paper. The 'health field concept' basically work on the assumption that four crucial factors that influence health, i.e. socio-economic, cultural and physical environment, genetic (biology), individual lifestyles and health services must be tackled concurrently through preventive health education to achieving total health. However, according to Tones \& Tilford (1994), the preventive health product while is being promoted by health education is frequently intangible and offers, gratification at some indeterminate time in the often distant future; Implying that providing information and education to create awareness increase knowledge and positively induce a relatively permanent change in attitudes, behaviour and practices have a time and decision making dimensions, that Preds behavioural matrix and Haggestrands information diffusion theory might provide further explanations.

Employing the concept of 'Satisfaction and bounded rationality' (Gregory, 1985), Pred depicted decision-making outcome as a function of 'quality and quantity of information available in a given situation and the individual decision maker's ability to handle the information'. His model recognized

West African Journal of Applied Ecology - Volume 13 
four factors as influencing individual's ability to use information, namely the users experience, intelligence, flexibility and adaptability to new information; and desire, preference, attitude and expectations. In other words, a person can have perfect knowledge about something, but the utilization of such information may be minimal or poor, and others may have little knowledge, but may make optimum use of it; such individuals he termed 'rational beings'.

Complimenting Preds model, the diffusion theory explains why, in practice, behavioral and attitudinal changes occur slowly, or may not happen at all with some people in any given social context. According to Hagestrand, theoretically the spread of phenomena over space through time interact in a matrix of contours which structures the way information circulates through a regional system. The flow of ideas are modulated by both physical barriers and individual resistances, which together check the transformation of information into innovation and so shape successive diffusion waves that breaks into adoption surfaces (Johnston, Gregory \& Smith, 1998).

The diffusion concept basic assumption is that initially when information is made available only a few people grasp the essence of the new idea or innovation and modify their behavior in response to new events in the environment (Awusabo-Asare, 1998). As indicated in Fig. 3, when information is diffused, the first groups of people to accept are known as 'innovators' (16\%). They form a small group of the population. This is followed by 'the early majority' (34\%), 'the late majority' (34\%) and finally 'the laggards' $(16 \%)$. The latter are those who, for one reason or the other, are resistant to the innovation. The pattern of adoption follows a logistic curve as Fig. 3 shows. The rate of adoption, however, depends on a number of factors including the nature of the innovation, the opportunity costs of the change, location and the socio-cultural, environmental and psychological factors that may hinder or promote the spread of the innovation. These concepts would inform analysis of stakeholders' perception of heath education situation in Ghana.

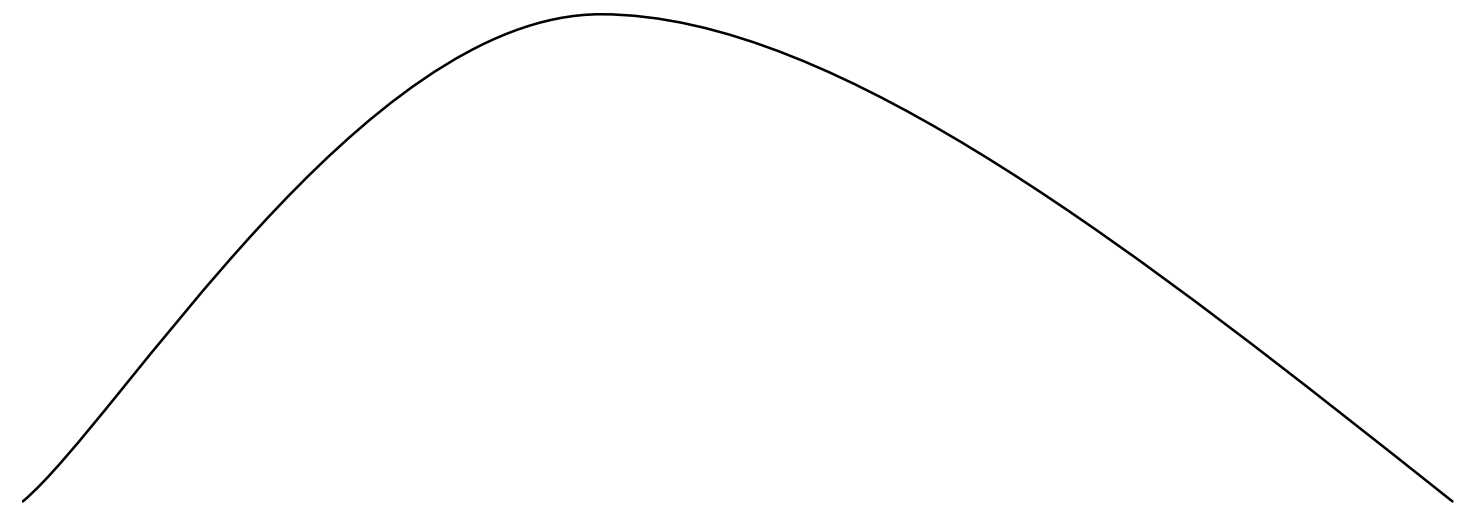

Source: Johnston, Gregory \& Smith, 1998

Fig. 3. Theoretical structure of Hagestrand's (1953) Diffusion of Information Model

\section{Background to Ghana's health education program}

Ghana was a signatory to the 1978 Alma-Ata Declaration and adopted the PHC philosophy as a policy for its health care system. This was made immediately possible due to a successful implementation of the Brong-Ahafo Rural Integrated Development Program (BARIDEP), which started in 1975 (MOH, 1993). A total of 31 community clinics were in place under the BARIDEP project by 1978 when the PHC policy was adopted. This was a pilot program aimed at obtaining experience and technical knowledge that could enable similar projects to be replicated in other areas of the country.

Under the auspices of the Ministry of Health (MOH), Ghana's PHC ultimate policy goal has been to promote good health among all citizens through 'preventive' and 'curative' health services, using the West African Journal of Applied Ecology - Volume 13 
appropriate form of technology and involving the people. To achieve this, two policy objectives were realized by the end of 1990 . These were to provide basic PHC coverage for $80 \%$ of Ghanaians, and to effectively attack all diseases that contribute to $80 \%$ of avoidable deaths and disabilities among Ghanaians. Strategies designed to meet the above objectives include: (1) Decentralizing the administrative and management of procedures of the health ministry; (2) Organizing massive health education programmes within all communities, work places and in the homes in cooperation with agencies such as the Ministries of Youth and Rural Development, Labour and Social Welfare, Education and Agriculture; (3) Promoting maternal and child health services, including family planning and nutrition, expanded programme of immunization (EPI) to overcome the very high morbidity and mortality in the children population of the six killer diseases such as measles, poliomyelitis, whooping cough, diphtheria, tuberculosis and tetanus $(\mathrm{MOH}, 1993 ; 1996)$. These strategies are being run on a three-tier system of care; from level A (Community), Level B (Health Centre/Post, i.e. sub-district) to level C (District Hospital). These structures incorporate a functional referral system that is very crucial to give real meaning to the interface of the level (Dennis, 1992).

According to the various policy papers reviewed from the health ministry (MOH, 1995, 1997, 2001), to accomplish the policy objectives set above 'health education' as an intervention strategy has been implemented as a support activity through the: (1) Production of materials known to be effective in sufficient quantities and distributed widely; (2) Strengthening the use of Radio and TV to educate the public; (3) Training of regional health education officers in message and materials development to use local media such as drama groups, associations and individuals for effective education; (4) Involvement of communities and other relevant sectors in the development and implementation of health education programmes, including IEC training; and (5) Directing health education activities to emphasize specific priority health issues, especially family planning, disease control, immunization, malaria, acute respiratory infections, diarrhoea; environmental sanitation, nutrition, oral and mental health, and campaigns for healthy life styles.

In theory, health promotion and education activities and delivery at the district level should be carried out through inter-sector collaboration. This implies health care providers working closely with other human development professionals in the district environmental health, social welfare, water and the agricultural extension units. In practice, however, this had hardly been the case to help in the realization of total health.

On the whole, Ghana has made some gains in health outcomes, albeit slowly, given that life expectancy at birth has increased from 47 in 1970 to 58 years in 1998. However, according to a Report of Health and Disease Analysis Task Team (MOH, 2001), there are still deprivations in many respects. For instance, '20 years ago, malaria, measles, childhood pneumonia, sickle cell disease, severe malnutrition, premature death, birth injury, accidents, gastroenteritis and tuberculosis collectively accounted for $57 \%$ of the total burden of disease in Ghana, as measured by healthy days of life lost through illness, disability and death' and this has not changed.

What is strikingly disturbing is the fact that the pattern of the leading causes of outpatient morbidity reflects environmental ill health. About $70 \%$ of the economic cost of health problems in Ghana has been attributed to environmentally related diseases, taking account of labour and the costs of resources (Leitmann, 1992; MOH, 2001). The implication of these realities is that the modest achievement of health tends to fall far short of the targets set as far back as 1978 when the PHC policy was adopted, hence, the need to look at the contributions made, and continued to be made, by health education from stakeholders' perspectives in the nation's search for improve health in a cost-effective manner.

\section{Materials and methods}

The paper is based on a cross-sectional survey design carried out in 1997 from stakeholders of public health care service delivery in Ghana (i.e. policy makers, health care service providers and the public/users of health care). The purpose of the study was to explore the status of health education under Ghana's PHC program and how they perceive it.

West African Journal of Applied Ecology - Volume 13 
Gilbert (1993) has suggested the need for social research to be located in an academic discipline. Consequently, this paper's foundation is based on a micro-survey contextualized in health promotion or prevention context. Additionally, Gilbert considered theory construction, data collection and design of methods for gathering data as three major elements in social research which must all be right if the results of study should be interesting and of some value. Having dealt with the former, therefore, this section focuses on the design of methods for collecting data.

\section{Data sources}

Data were derived from both secondary and primary sources. Prominent among the former are published and unpublished literature on he subject, the 1998 and 1993 GDHS; various health policy papers, including MOH (2001); The Health of the nation (2001), MOH (1996), Medium Term Health Strategy 1996-2000, MOH (1996), 5-Year Programme of Work, MOH (1994/95); Policies and Priorities, the 1984 Ghana Population Census Report, the 1998 Human Development Report, and WHO First International Conference on Health Promotion. The latter was collected from interviews done with health policy makers, managers, service providers and the public.

\section{Sampling and data collection procedures}

The study was designed basically as a descriptive qualitative approach that employed non-probability sampling techni-ques to select the study population. The convenient method was used to select policy makers and health managers at national and regional levels, and interviewed using semi-structured interview schedule. Quota sampling method was employed to select 64 different categories of health care providers in Cape Coast, as shown in Table 1.

TABLE 1

Selected healthcare providers interviewed $(n=64)$

$\begin{array}{lrlrl}\begin{array}{l}\text { Curative health providers } \\ \text { (PHCP) }\end{array} & \begin{array}{l}\text { No. } \\ \text { inter- } \\ \text { viewed }\end{array} & \begin{array}{l}\text { Preventive health providers } \\ (\text { PHCP })\end{array} & \begin{array}{l}\text { No. } \\ \text { inter- } \\ \text { viewed }\end{array} & \begin{array}{c}\text { Total } \\ \text { Enroll Nurse }\end{array} \\ \text { SRN General } & 3 & \text { Community Psychiatric Nurse } & 3 & 6 \\ \text { Staff Nurse Midwife } & 3 & \text { Community Health Nurse } & 3 & 6 \\ \text { Psychiatric General Nurse } & 3 & \text { Public Health Nurse } & 3 & 6 \\ \text { Nurse Anaesthetic } & 3 & \text { Nurse Educators (Tutors) } & 3 & 6 \\ \text { Medical Doctors (Clinicians) } & 2 & \text { Medical Doctors (Public Health) } & 3 & 5 \\ \text { Medical Assistant } & 3 & \text { Disease control Technicians } & 3 & 6 \\ \text { Pharmacist } & 3 & \text { Disease Control Field Technicians } & 3 & 6 \\ \text { Dispensing Technicians } & 3 & \text { Environmental Health Technicians } & 3 & 6 \\ \text { Laboratory Technicians } & 3 & \text { Environmental Health Field Officers } & 3 & 6 \\ \text { X Ray Technicians } & 3 & \text { Health Educationist } & 3 & 6 \\ \text { Blood donor organiser } & 1 & \text { Bio Statistician } & 1 & 2 \\ \text { Total } & 2 & \text { Nutrition Technical Officer } & 2 & 4 \\ & 32 & \text { Total } & 32 & 64\end{array}$

Source: Cape Coast Field Survey, April 1997

The rationale for this sampling strategy was to achieve some kind of balance with the representation of both curative and preventive health professionals and their views, generally on healthy education as a

West African Journal of Applied Ecology - Volume 13 
programme activity in the health sector. The health personnel were interviewed using structured questionnaire administered personally by the author, and collected within 10 days. Issues covered include, among others, equipment and logistic capacity, human resource, pre and post service training, current impact of health education, operational focus of health education; and effective medium of health education delivery.

With the public (i.e. users of health care), focus group discussions (FGDs) were purposively carried out in three different health education delivery settings, namely Pedu JSS in Cape Coast (involving four girls and four boys in JSS 3, average age 16 years); Siwudu Garage Workshop in Cape Coast (made up of eight male apprentices between 18 and 34 years); and Ntronoa Community in KEEA district (involving four males and three females, average age 22 years). The discussions lasted about 45 min and focused generally on benefits of health education, sources of information on health, family life and sex education, public attitude to refuse disposal, content of health messages, first aid and major health problems in their community.

\section{Limitations to data collection}

Since health education is only seen as providing a 'supportive role' to achieving the objectives of $\mathrm{MOH}$ priority programmes, quantitative data to enhance the investigation at all levels was not available. Additionally, and, perhaps more problematic, was the ethical issue of the perception that curative health care professionals are often hostile to preventive health programmes, hence, including them in the sample selection might not help in the objective assessment of the subject matter. Finally, although data were collected from all levels of health activity, interpretation had to be done with caution so as not to generalize the results as representing the overall national picture.

\section{Results}

The section briefly but succinctly assesses the state and impact of health education as an integral component of the PHC programme using both secondary and primary data indicated earlier. It is presented in three perspectives by examining national health education policy and programmes of activities from human, material and financial points of view; assessing current situation of health education to health promotion as seen by the stakeholders; and discussing the important issues that emerge based on the lessons and implications within the context of the theoretical perspectives.

\section{Health education policy thrust}

Analysis of secondary data including a 1990 PHC review by Adjei (1991) and the 1994 and 1996 Health Policy guidelines suggests that the health education unit of the MOH has been in place since 1968. There was evidence of policy support in the two policy documents, emphasizing the need to intensify health education aimed at fostering healthy lifestyles through provision of technical assistance on health education to various programme delivery units and health related agencies. Yet, there was not enough evidence of past or present documented plan of action, specifying programme direction and resource commitment to this (Obeng-Quaidoo et al., 1992). The only evidence, which was quite anecdotal, related to the 1988-1991 Information Education and Communication and Family Planning (IEC/FP) pilot project launched in the three regions of Ashanti, Brong-Ahafo and Central.

\section{Equipment and logistic capacity}

Regarding equipment and logistic support to the health education programme at the policy level, it was found that it is probably the least resourced unit in the health ministry. For example, from the national to the district level, available and equipped vehicles for programme support were 10 Landover's supplied as part of the 1989-90 IEC/FP project, and allocated to the regional health education units. It is difficult to understand how effective health education can be planned, organized, supervised and monitored 
adequately even in a relatively smaller region as Greater Accra, let alone Northern Region, given its geographical dimension with a single vehicle. This observation tends to belittle the definition of health education given by Tones \& Tilford (1994).

\section{Human resource capacity}

Available secondary data did further suggest that by public health policy practice, the $\mathrm{MOH}$ has maintained health education professionals at only national and regional levels. Hence, with one professional health educator in 1957, there were only 16 by 1997, providing a ratio of one health educator to over a million Ghanaians. This policy is based on the assumption that every health care professional is a health educator. However, data from the health providers interviewed, and indicated in Table 2, show that about $80 \%(79.7 \%)$ had some partial training in health education skills during their pre-service training.

TABLE 2

Pre and post service training of respondents $(n=64)$

\begin{tabular}{|c|c|c|c|c|c|c|}
\hline \multirow[t]{2}{*}{ Type of training } & \multicolumn{2}{|c|}{ Curative health providers } & \multicolumn{2}{|c|}{ Preventive health providers } & \multicolumn{2}{|l|}{ Total } \\
\hline & Yes & $\mathrm{No}$ & Yes & No & Yes & No \\
\hline $\begin{array}{l}\text { HE inclusive in pre- } \\
\text { service curricular }\end{array}$ & $26(40.6 \%)$ & $6(9.3 \%)$ & $25(39 \%)$ & $7(10.9 \%)$ & $51(79.7 \%)$ & $13(20.3 \%)$ \\
\hline $\begin{array}{l}\text { Adequately equipped to } \\
\text { educate/ inform others }\end{array}$ & $19(29.7 \%)$ & $13(20.3 \%)$ & $22(34.4 \%)$ & $10(15.6 \%)$ & $41(64 \%)$ & $23(35.9 \%)$ \\
\hline $\begin{array}{l}\text { Post-service training in } \\
\mathrm{HE} / \mathrm{IEC}\end{array}$ & $18(28 \%)$ & $14(21.9 \%)$ & $24(37.5 \%)$ & $8(12.5 \%)$ & $42(65.6 \%)$ & $22(34.4 \%)$ \\
\hline $\begin{array}{l}\text { Post-service in guidance } \\
\text { counselling }\end{array}$ & $17(26.5 \%)$ & $15(23.4 \%)$ & $13(20.3 \%)$ & $19(29.7 \%)$ & $30(46.9 \%)$ & $3453.1 \%)$ \\
\hline
\end{tabular}

Source: Authors field survey, April 1997

Although, over $64 \%$ of them perceived they are equipped enough to impart information and knowledge to others, this has to be interpreted with caution given that the providers consulted were urban based (in Cape Coast), the capital of Central Region. Because it has been observed that, in relative terms, the best professional personnel tends to be allocated to the urban areas, which has more of the well equipped hospital facilities, whether by accident or policy design. The investigations also revealed that it was in 1988-91 that about 5000 health care providers from the three FP pilot campaign regions mentioned earlier were trained in IEC/FP skills to enable them give effective health education. It came out also that in the early 1990s the HIV/AIDS menace did spur the National Aids Control Programme (NACP) to encourage health education through the training of selected providers in counselling and IEC skills (Barnet et al., 1995).

\section{Financial capacity}

Evidence gathered from the national health ministry tend to show that whereas the health policies reflect a shift in emphasis from curative to preventive health, the national health budget allocates about $70 \%$ of the total budget to curative services (MOH, 1995; Kunfah, 1996; Ofosu Armaah, 1975). For the health education programme, planned financial resource allocation has been increasing, though marginally, over the years. However; the actual allocation has averaged about $0.22 \%$ of total actual allocations between 1992 and 1996 as shown in Table 3.

West African Journal of Applied Ecology - Volume 13 
TABLE 3

Financial allocation to health education programme (in billions)

$\begin{array}{llllll}\text { Sectoral allocation } & 1992 & 1993 & 1994 & 1995 & 1996 \\ \text { Programmed allocation to HE } & 0.197(0.7 \%) & 0.595(1.3 \%) & 0.655(1.4 \%) & 0.650(1 \%) & 0.970(1.1 \%) \\ \text { Actual allocation to HEP } & 0.049(0.2 \%) & 0.194(0.6 \%) & 0.211(6.4 \%) & 0.254(0.4 \%) & 0.119(0.1 \%) \\ \text { Total actual MOH allocation } & 24.3 & 96.9 & 52.7 & 63.5 & 118.6\end{array}$

Source: MOH, Annual Budget Estimates, 1991-1996

\section{Current and potential effects of health education}

Results of the interviews with the health care providers supported the literature that in practical terms four appropriate settings of HE delivery exits. These include, reaching individuals, families and groups in communities, schools, workplaces and health institutions as in Table 4. However, the reality is that, generally, health education has been inadequate at all levels, except at static health facilities, i.e. hospitals, health centres/posts, MCH/FP centres, Clinics, where more than half $(56 \%)$ of the on-going health education activity tend to take place as Table 4 shows. It was indicated, however, that at the institutional level the information delivery strategy has been the routine, and sometimes unimaginative 'one-way health talks', given to the sick or psychologically less prepared people attending to their sick ones.

TABLE 4

Operational focus of health education ( $n=64)$

HE delivery setting

Level of adequacy ranked by health care providers Curative health providers Treventive health providers Total

Schools $\quad-0.19 \quad-0.01 \quad-0.20$

$\begin{array}{lll}\text { Health Institutions } & +0.23 & +0.33\end{array}$

$\begin{array}{llll}\text { Workplaces } & -0.34 & -0.36 & -0.70\end{array}$

$\begin{array}{llll}\text { Communities } & -0.2 & -0.03 & -0.05\end{array}$

Source: Cape Coast Field Survey, April 1997

[Survey scale: Very Adequate (1), Normal (0), Inadequate (-1), Very Inadequate (-2)]

The respondents agreed to a question that one might be right to describe health education towards prevention of ill health as ad hoc. Because, according to them, health education is only intensified during disease or epidemic outbreaks, or when there is national/international day, such as World Aids Day to commemorate. One of the principal reasons accounting for this was found to be inadequate personnel, reenforced by lack of motivation to health care providers, under the unproven policy assumption that everyone is a health educator. Results from the provider interviews and the FGDs with the public revealed that the three most effective media of health education delivery are the person-to-person or one-on-one approach (67\%), followed by television (11\%) and radio (10\%) as presented in Table 5.

TABLE 5

Health providers perception about the effective medium of health education delivery $(n=64)$

Type of medium

Level of effectiveness ranking

Curative health providers Preventive health providers Total

West African Journal of Applied Ecology - Volume 13 


$\begin{array}{lccc}\text { Television } & -0.05 & +0.16 & +0.11 \\ \text { Leaflets/Magazines/Newsletters } & -0.03 & -0.05 & -0.08 \\ \text { Video } & -0.61 & -0.01 & -0.62 \\ \text { Radio } & +0.23 & +0.27 & +0.50 \\ \text { Cinema } & -0.43 & +0.09 & -0.34 \\ \text { Person to person } & +0.05 & +0.62 & +0.67\end{array}$

Source: Cape Coast field survey, April 1997 [Survey scale: Very effective (2), Effective (1), Ineffective (-1), Very ineffective (2)]

The low ratings of the print media have two implications. First, the people generally do not enjoy reading because nearly all the newspapers and other magazines are published in the English language, but most people, especially from the rural areas cannot read or do not have easy access. Secondly, the mass media (especially the newsprint) tend to give health issues less priority. One can infer that this is probably due to the fact that media practitioners, in general, have not been adequately involved in health matters to enable them to report and interpret issues so as to promote healthy decision-making and choice among the populace. Results of the FGDs and interviews with the health care providers tend to show that the people generally share the opinion that ineffective and, perhaps, poorly coordinated health education programme has, to a large degree, contributed to the poor public attitude and behaviour towards health and environmental sanitation in many Ghanaian communities. They also added that many other factors contribute to the present situation of health education and the response of the public to information received. Reacting to the non-adoption of diet and nutrition education practices people have heard from health care providers, it came out from the Ntronoa community FGDs that both children and adult malnutrition (Kwashiorkor) is due to poverty. The impression one gets from the respondents is that poverty appears to have somehow contributed to the issue of sheer carelessness and moral laxity, leading to insensitivity of Ghanaians towards health education.

\section{Discussion}

The paper has attempted to highlight the status of health education in Ghana from the purview of various stakeholders. The views expressed were not just concerned with a descriptive fact gathering exercise, but also with understanding the social context of the actors involved. Analysis of the views from all stakeholders tends to agree that ignorance and lack of adequate and sustainable information flow, as argued by Hubert (1994), has contributed to the relatively poor health of the Ghanaian population. The respondents' view that poverty, rather than lack of information, affects health supports Kunfah's (1996) observation about the inseparable relationship between health and other crisis factors. For example, poor economic performances of many SSA countries have affected the spending of the people on improving health and welfare. It can also be argued that low income, one of the crisis factors identified by Kunfah (1996) as affecting health, could be contributing to the under-funding of health care and the health education activities at all levels of health delivery system in Ghana.

Health policy-makers, managers and providers supported the view that fiscal, human and logistical limitations has, to a large extent, constrained the health education programme. However, it is interesting and worthy of note that users of health care services disagreed with Hubert (1994) that children and adult malnutrition is not mainly due to ignorance or lack of awareness, but poverty has, to a greater extent, contributed to the health seeking behaviour of the Ghanaian.

The perception that health education programmes and activities tend to be ad hoc, one-way information flow (mainly at health care institutions) and under resource is not good enough, and tends to make mockery of the health promotion concepts proposed by WHO $(1986 ; 1984)$, and supported by Tannahill et al. (1990) and Ewles \& Simnett (1992).

Lack of adequate post service training on 'Health Education' to providers of health, coupled with unsustainable health education delivery to users of health care is contrary to the time and decision making

West African Journal of Applied Ecology - Volume 13 
dimensions of the Hagastrand information diffusion and Preds behavioural theories (Johnston, Gregory \& Smith, 1998; Gregory, 1985). Furthermore, it tends to ignore Tones \& Tilford (1994) observation that the product being propagated by health care not to despair, but to persist if their efforts seemed not to be yielding the expected results on time, since that is practically in line with the natural behaviour of the population in a given social context.

The respondents' view that person-to-person, radio and television education are the most effective medium of health education delivery has challenging resource implications to the Ghana Health Service (GHS), particularly the professional personnel to implement the first strategy for instance. The radio and television rating partly support Haryono's (1996) analysis of the cross-country survey reports from Botswana, Mauritius, Malaysia, Singapore and Indonesia, where the mass media, especially radio and television, has been used effectively and quite successfully to educate the public. He observed that through public policy support the six different television stations in Indonesia carry a mandatory 2-h IEC programme towards health promotion, with emphasis on population control and family planning. This is something worth considering in Ghana, given similar socio-cultural background.

\section{Conclusion}

This paper has examined health education situation in Ghana from the purview of the various stakeholders. It can be argued that health and illness will continue to occupy a prominent place in Ghana. Hence, there is the need to open up to the age old saying that prevention is better than cure, because that is the philosophical principle behind health education as social activity. This implies that from both theoretical and practical point of view, health education is a worthwhile activity and service that can bring about health promotion over a long term as a process activity.

From the findings and discussion it can be said that health education is an integral component of Ghana's health care delivery system and needs to be re-asserted and recognized as such from policy to the community level, where routine or periodic activity takes place. In this regard, besides financial resource commitment to support health education activities genuinely, it is important to equip the service providers with the technical skills and knowledge through periodic training, thereby, preparing them adequately to provide all stakeholders with the information required to improve their health seeking behaviour. This is necessary because both the diffusion and behavioural theories thrive on quality information delivered in timely fashion to inform decision-making.

It can be argued from the Lalonde's health field concept that health education activities at the local level should be based on healthy inter-sectional collaboration, by involving decentralize departments such as education, agriculture extension, community water and sanitation, environmental health and social welfare, all of which have realistic contributions to make towards health protection and prevention as advocated by Tannahil (1994). Based on the lessons from Botswana, Mauritius, Malaysia, Singapore and Indonesia, there is the need for a sustained policy and action to effectively tap the potential of both the public and private media resources to promote health through regular education. For instance, the GHS needs to consciously make use of the numerous FM stations, in particular, to promote health and wellbeing.

More empirical research is needed, especially on perceived and actual benefits of health education in different cultural settings and among social groups, to enhance the understanding of the indispensable part health education plays, and will continue to play in the health delivery system, for many years to come.

\section{Acknowledgement}

Many individuals have been of invaluable help in the preparation of this paper. The author is indebted to Dr E. Y. Kunfah of the Department of Planning, Kwame Nkrumah University of Science and Technology for supervising the initial research. He expresses sincere thanks to all the respondents from both the Ministry of Health and the community members, who provided the primary data. The motivation, encourage-ment, and support to get the work published has come mostly from senior colleagues of the West African Journal of Applied Ecology - Volume 13 
Department of Geography and Tourism, namely Prof L. E. Dei, Prof. K. Awusabo-Asare, Prof Jurly Blankson, Dr A. M. Abane, Dr Ohenba Acheampong and Mrs Dellali Badasu. He is equally grateful to his friend and colleague, Dr E. K. Ekumah of the Centre for Development Studies, University of Cape Coast (UCC), for sustaining his interest in health policy. Finally, sincere thanks go to Mary Aprekuwah Osapah of the JICA Supporting Unit, UCC, for her selfless secretarial support in putting the pieces together.

\section{References}

Adjei S. (1991). Review of PHC in Ghana. Ministry of Health, Accra.

Asamoah-Baah A. (1990) Primary Healthcare in the 1990's: Concepts and Challenges. Paper presented at the meeting of the Director General's Consultative Committee for PHC Development, Geneva, 9-12 April 1990, pp. 1-9.

Awusabo-Asare K. and Anarfi J. K. (1999). Routes to HIV Transmission and Intervention: an analytical framework. Paper presented at the meeting of the West Africa Research Group on Sexual Networking (WARGSN) held at the University of Ibadan, Nigeria, 4-14 December 1997.

Collins C. (1994). Management and Organisation of Developing Health Systems. Oxford University Press. Oxford.

Dennis J. A. (1992). Health promotion or Education and the New Public Health: Their Role in the Healthcare System in Ghana, (MSc. Dissertation) Department of Public Health, University of Edinburgh.

Ewles L. and Simnett I. (1992). Promoting Health: a practical guide, 3rd edn. Scutari, London.

Friedman J. (1992). Empowerment: The Politics of Alternative Development. Blackwell, Oxford and Massachusetts.

Gilbert N. (1993). Researching Social Life. Sage Publications, London.

Green L. W. and Raeburn J. M. (1988). Health Promotion, What is it? What will it Become? J. Hlth Prom. 2: 151-158.

Gregory D. (1985) Suspended Animation: the stages of diffusion theory. In Social Relations and Spatial Structures. (D. Gregory and J. Urry, ed.) Macmillan, London.

Hagget P. and Chorley R. J. (1968) Socio-Economic Models in Geography. Methuen, London.

Haryono S. (1996). Indonesia, Prosperous Family Development. In International Review of Population and Reproductive Health. Integration 49: 27-32.

Hubert B. (1994). Health in the Developing World: Progress, Despite Everything. The Courier 147: 47-50.

Johnston R. J., Gregory D. and Smith D. M. (1998). The Dictionary of Human Geography, 3rd edn. Blackwell Publishing Inc., Oxford.

Kelly M. P. (1990) World Health Organisation Definition of Health Promotion: Three Problems. Hlth Prom. Bull. 48(4): 17-30.

Kok G. and Green L. W. (1990). Research to Support Health promotion in Practice: A Plea for Increasing Cooperation. Hlth Prom. Int. 5(4): 3030-308.

Kunfaa E. Y. (1996). Sustainable Rural Health Services through Community-based Organiza-tions: Women's Groups in Ghana. Spring Research Series No. 16. University of Dortmund.

Lalonde M. (1974). A New Perspective on the health of Canadians. Government of Canada, Ottawa.

Lee K. and Mills A. (1983). Health and Health Environment in the Economies of Health in Developing Countries. Oxford University Press, Oxford.

Leitmann J. (1992). Environmental Management and Urban Development in the Third World: A tale of Health, Wealth and the Pursuit of Pollution from Four Cities in Africa, Asia, Eastern Europe and Latin America. (PhD Thesis.) University of California, Berkeley. Cited in Better Health in Africa: Experiences and Lessons Learned. World Bank, Washington DC.

M'Jamtu-sie M. (1996). Health Information for the Grass Roots. Int. J. Hlth Dev. 17(3): 277-283.

Ministry of Health (1993). MCH/FP Annual Report. Ministry of Health, Accra.

Ministry of Health (1995). Medium Term Health Strategy: Towards Ghana's Vision 2020. Ministry of Health, Accra.

Ministry of Health (1996). Medium Term Health Strategy. Ministry of Health, Accra.

Ministry of Health (1997). Road to Healthier Future for Ghana. Ministry of Health, Accra.

Ministry of Health (2001). Reflections on the First Five-Year Health Sector Programme of Work, 1997-2001, Ministry of Health, Accra.

Ministry of Health (2001). Report of Health and Disease Analysis Task Team. Ministry of Health, Accra.

Ministry of Health (1989). Health Education Unit Annual Report. Ministry of Health, Accra.

Ofosu-Armah S. (1975). Reflections on the Health Budget. Ghana med. J. 14: 215.

Tannahill A., Downie R. S. and Fyfe C. (1990). Health Promotion: Models and Values. Oxford University Press, Oxford.

Tones K. and Tilford S. (1994). Health Education: Effectiveness Efficiency and Equity, 2nd edn. Chapman and Hall, London.

World Health Organization (1988). Education for Health: A Manual on Health Education in Primary Health Care. WHO, Geneva.

World Health Organisation (1984). Strengthening Ministries of Health for Primary Health Care. Geneva, WHO.

World Health Organisation (1986b). First International Conference on Health Promotion. Ottawa Charter, Canada. WHO, Geneva.

West African Journal of Applied Ecology - Volume 13 
World Health Organisation (1986c) International Action for Health: The Role of Inter-Sectoral Cooperation in National Strategies for Health. WHO, Geneva.

World Health Organisation (199d). Development 1997, WHO Programs and Activities. WHO, Geneva.

West African Journal of Applied Ecology - Volume 13 